Original Article

\title{
Endovascular Deep Vein Stenting of Symptomatic Post-Thrombotic and Non-Thrombotic Iliac Vein Stenotic Lesions: A Multicentre Cohort Experience from Singapore
}

Mervin Nathan Lim Han Hui, ${ }^{1}$, Karthikeyan Damodharan, ${ }^{2}$ MBBS, FRCR, Sze Ling Chan, ${ }^{3}$ PhD, Ming Ren $\underline{\text { Toh }},{ }^{4} M B B S$, Charyl Yap Jia Qi, ${ }_{B S C}$, Tze Tec Chong, ${ }^{4,5}$ MBBS, FACS, Tjun Yip Tang, ${ }^{4,5}{ }_{M D}$, FRCS

\begin{abstract}
Introduction: This paper presents our experience with deep venous stenting in a multi-ethnic Asian cohort of patients with symptomatic Non-Thrombotic Iliac Vein Lesions (NIVL) and Post-Thrombotic Syndrome (PTS). Materials and Methods: This was a multicentre retrospective cohort study of patients who had symptomatic deep venous disease. Stent patency rate was evaluated using Duplex ultrasonography immediately post-intervention and at 3, 6 and 12 months. Clinical outcomes were evaluated using the revised Venous Clinical Severity Score (rVCSS) and Visual Analogue Scale (VAS) pain score at baseline and 3 months post-procedure. Results: 87 patients $($ males $=47 / 87(54.0 \%)$ ); median age $=62$ years $($ IQR $55-70)$ ) and 115 limbs were analysed $($ left $=76 / 115(66.1 \%))$. Median follow-up time was 175 (IQR 57 - 257) days. 97/115 (84.3\%) had NIVLs and 55/115 (47.8\%) had May-Thurner-Syndrome. 43/115 (37.4\%) had Clinical, Etiology, Anatomy and Pathophysiology (CEAP) 6 disease. Primary stent patency rates were $\mathbf{9 8 . 2 \%}$ $(112 / 114), 97.9 \%(93 / 95), 95.7 \%(89 / 93)$ and $92.8 \%(64 / 69)$ immediately postintervention, 3, 6 and 12 months, respectively. The 6-month secondary patency rate was $99.1 \%(114 / 115)$. Mean rVCSS and VAS improved from $11.52( \pm 3.54)$ to 5.77 $( \pm 2.36)(P<0.01)$ and $6.62( \pm 1.93)$ to $2.92( \pm 1.50)(P<0.01)$ respectively, at 3 months. $41 / 43(95.3 \%)$ venous ulcers healed over a median time of 169 days (IQR 120 - 253). Conclusions: Short term primary patency rates following deep venous stenting are excellent, with few re-interventions. Patients presented with NIVLs rather than PTS. There was excellent clinical improvement at 3 months, with a high and expedient venous ulcer healing rate.
\end{abstract}

Ann Acad Med Singapore 2020;49:551-60

Key words: Deep vein stenting, May-Thurner Syndrome, Non-thrombotic iliac vein lesion, Post-thrombotic syndrome, Vascular patency

\section{Introduction}

Chronic ilio-femoral venous obstruction (IFVO) is a debilitating disease. Symptoms include venous claudication, swelling and venous stasis ulcers. ${ }^{1}$ Non-thrombotic iliac vein lesions (NIVL) and post-thrombotic syndrome (PTS) can cause IFVO. NIVL includes May-Thurner Syndrome (MTS), where the left common iliac vein (CIV) is compressed between the right common iliac artery and the 5th lumbar vertebra. ${ }^{2}$ Right sided and bilateral MTS have also been reported, albeit rare. ${ }^{3-5}$ PTS develops in up to half of patients with deep vein thrombosis (DVT), causing intraluminal scarring and venous obstruction. ${ }^{6}$ Previously, IFVO was treated with analgesia, compression stockings and anticoagulation. Bypass surgery was done for severe cases but results were guarded and is not considered to be first line treatment. ${ }^{7}$

Recently, percutaneous deep vein stenting has been shown to be an effective treatment modality for such lesions, regardless of aetiology. ${ }^{8}$ Dedicated venous stents

\footnotetext{
'Yong Loo Lin School of Medicine, National University of Singapore, Singapore ${ }^{2}$ Department of Interventional Radiology, Sengkang General Hospital, Singapore ${ }^{3}$ Health Services Research Center, SingHealth, Singapore

${ }^{4}$ Department of Vascular Surgery, Singapore General Hospital, Singapore

${ }^{5}$ Duke NUS Graduate Medical School, Singapore

Address for Correspondence: Dr Tjun Yip Tang, Department of Vascular Surgery, Singapore General Hospital, Level 5, Academia, 20 College Road, Singapore 169856

Email: tang.tjun.yip@singhealth.com.sg
} 
with high radial forces and flexibility are now used. ${ }^{7}$ Multiple studies have reported high technical success rates and positive clinical results. A meta-analysis conducted by Wang et al showed that endovascular stenting of IFVO was associated with low complication rates and desirable long-term patency rates. ${ }^{9}$ While existing studies show favourable clinical outcomes, most capture a vastly Caucasian demographic., ${ }^{70,11}$ There are few reported studies investigating the efficacy of deep vein stenting with intravascular ultrasound (IVUS) interrogation, and the epidemiology of IFVO in a multiethnic Asian background, such as that in Singapore. ${ }^{12}$ Most existing Asian studies investigated patients with computed tomographic venogram (CTV) or venography, without IVUS. The majority of these studies used venography to diagnose IFVO and place their stents, which also were not dedicated to the venous system. ${ }^{13-19}$ We have previously reported that truncal vein diameter, distribution of venous reflux, and symptomatology may differ between Asians and Caucasians. ${ }^{20}$

The deep vein stenting experience in Singapore is relatively immature with only a few centres performing this potentially quality of life (QoL)-changing procedure. To date, no local outcome data has been published. Singapore General Hospital (SGH) set up its deep venous program in April 2018, and has performed over 160 such procedures since.

The aim of this paper is to present our experience with deep venous stenting of IFVO, for symptomatic NIVL and PTS patients. Short-term clinical outcomes and stent patency rates of patients from a multi-ethnic Asian background in Singapore were evaluated.

\section{Materials and Methods}

\section{Patients and Study Design}

This was a multi-centre retrospective review of 87 patients (115 legs; 118 interventions) with symptomatic PTS or NIVL deep venous lesions, who underwent endovascular ilio-femoral stenting with IVUS from May 2014 to May 2019 (5 years) at 3 of our local public hospitals, under the care of the senior author (TYT) as the primary physician. The majority of patients had at least 6 months of follow-up. The indications for deep vein intervention were symptomatic patients with severe chronic venous insufficiency affecting their QoL. They were offered IVUS and stenting after secondary causes (cardiac, renal, hepatic) of lower limb oedema were excluded. Patients with CEAP classification $^{21} 4 \mathrm{a}$ disease status and above were included. Patients' case notes and electronic records were reviewed. Patient demographics, co-morbidities, pre-morbid functional status, CEAP classification, along with procedural, angiographic and follow-up data were retrieved. The severity of chronic venous insufficiency and pain score were evaluated by the $\mathrm{rVCSS}^{22}$ and a numerical VAS, respectively, pre- and post-intervention (3 months). The local Institutional Review Board approved this study (CIRB no. 2018/3150).

Pre-operatively, all patients had a contrast-enhanced $\mathrm{CTV}$ or magnetic resonance venogram (MRV) to not only look for iliac vein compression, but (more importantly) also rule out intra-abdominal masses that may cause extrinsic venous compression. Pre-operative Duplex ultrasonography (DUS) was performed to investigate the presence of concomitant superficial and deep venous reflux. Follow-up of stent patency was performed using DUS and was standardised at Day 1 post-op and at 3, 6 and 12 months after intervention. Clinical outcomes were evaluated by comparing the rVCSS and VAS pain score at baseline and at 3 months after intervention. Healing times of venous ulcers were also documented. All venous ulcers had 4-layer compression applied until their wounds healed.

\section{Procedure and Postoperative Care}

The procedure was performed with the patient under general anaesthesia or deep sedation in the supine position. The ipsilateral femoral vein was accessed in an antegrade fashion, under ultrasound guidance, typically in the mid-thigh region using an 18-gauge needle with an aspiration $5 \mathrm{ml}$ syringe. A 5 French $(\mathrm{Fr})$ vascular access sheath (GLIDESHEATH ${ }^{\mathrm{TM}}$, Terumo Medical, Tokyo, Japan) was inserted and digital subtraction venograms were performed to image from the femoral vein up to the distal inferior vena cava (IVC) in at least 2 orthogonal planes. The venograms helped to identify stenosis or occlusion of the iliac veins and the presence of any collateral draining veins, which may indicate a significant stenosis. The ilio-femoral segment was crossed using a combination of a $4 \mathrm{~F}$ Bernstein catheter $\left(\mathrm{Tempo}^{\mathrm{TM}}\right.$, Cordis, USA) and a 0.35 " hydrophilic angled guidewire (Glide wire $^{\mathrm{TM}}$, Terumo Medical, Tokyo, Japan) under fluoroscopy. The hydrophilic guidewire was exchanged for a 0.35 " stiff guide wire (HI-TORQUE Supracore ${ }^{\mathrm{TM}}$ Abbott Medical, USA) wire and the access sheath was upsized to a $10 \mathrm{Fr}$ (GLIDESHEATH ${ }^{\mathrm{TM}}$, Terumo Medical, Tokyo, Japan) to allow IVUS interrogation and stenting, if required. The IVUS catheter (Volcano $^{\mathrm{TM}}$, Philips Healthcare, Eindhoven, Netherlands) was introduced 
over the guidewire into the distal IVC under fluoroscopy guidance and a preliminary pullback scanning run was performed to get an overview of the ilio-femoral lesions. IVUS pullback was performed both over the guidewire and without it, as the senior author had come to appreciate, with experience, that one can potentially get vessel distortion using the stiff wire, making a potential venous stenotic lesion more pronounced than it actually is. The lesions were typically eccentric rather than concentric and hence, cross sectional areas of the distal IVC, proximal CIV, mid CIV, iliac confluence, proximal mid and distal external iliac vein (EIV) and common femoral vein (CFV) were measured using the IVUS. In our experience, IVUS provides the most accurate estimate of the severity and extent of the venous lesions as well as the diameters of the normal iliac veins, which are not appreciated by venography alone, and is essential for deep venous intervention procedures. During the procedure, full anticoagulation with unfractionated heparin was given, targeting an activated clotting time of around 250 to 300 seconds. Pre-dilatation was performed using an appropriately sized balloon (usually 16/18 x 40/60 mm Atlas balloon, BD Medical, USA) for CIV and $14 \mathrm{~mm} \times 40 / 60 \mathrm{~mm}$ balloon for EIV/CFV). Using combined IVUS and fluoroscopic guidance, stents were deployed across the affected segment with a premise of stenting from normal to normal venous zone with no spot stenting allowed. Most of these lesions were chronic in nature and venoplasty alone was not sufficient to treat the fibrotic lesions. ${ }^{23}$ We preferred using dedicated self-expanding venous stents $\left(\mathrm{Venovo}^{\mathrm{TM}}\right.$, BD Medical, USA; Sinus Venous ${ }^{\mathrm{TM}}$, Sinus Obliquus ${ }^{\mathrm{TM}}$ Optimed, Germany). In our practice, we prefer to use the oblique venous $16 \mathrm{~mm}$ diameter stents (Sinus Obliquus $^{\mathrm{TM}}$, Optimed, Germany) for proximal CIV/MTS lesions as it provides the advantage of adequate coverage of the lesion without the risk of covering the contralateral CIV origin. We performed routine post dilatation of the stents with the appropriate sized balloons to achieve complete expansion of the stents. Venograms and IVUS were performed post-stent deployment to confirm adequacy of treatment, blood flow and for potential complications. The cross-sectional areas of the various segments of the veins were recorded and documented pre- and post-stenting. The access sheath and wires were removed and haemostasis was achieved with manual compression for 10 minutes without requiring any compression dressing or closure device.

Patients were placed in intermittent pneumatic compression postoperatively (Flowpac; Huntleigh Healthcare, Cardiff, UK) to promote flow through the stented segments of the vein. Therapeutic low-molecular- weight heparin (enoxaparin) was administered the same evening, followed by conversion to either warfarin (target international normalized ratio 2.0- 2.5) or new oral anticoagulants (rivaroxaban $20 \mathrm{mg} / \mathrm{d}$ ). For NIVL patients, anticoagulation was switched to aspirin $(100 \mathrm{mg} / \mathrm{d})$ or clopidogrel $(75 \mathrm{mg} / \mathrm{d})$ if DUS was satisfactory at 6 months. Those with PTS were either left long-term on $\mathrm{NOAC} /$ warfarin or switched to an antiplatelet agent after 1 year, depending on their thrombophilia status.

\section{Outcome Definitions}

Technical success was defined as successful deployment of stents to their intended locations. Procedural success was defined as technical success with at least one indicator of hemodynamic or clinical success. Stents were defined as patent when the DUS showed antegrade flow and a spontaneous Doppler signal, with maximal luminal stenosis of $£ 50 \%$. Primary patency rate was defined as the percentage of patients with uninterrupted stent patency until reintervention was required or in-stent thrombosis occurs. Secondary patency rate was defined as the percentage of patients with stent patency after primary procedural and technical success, irrespective of interval therapies.

\section{Statistical Analysis}

Continuous numeric variables were reported as mean and standard deviations for parametric distribution, and median (interquartile range (IQR)) for non-parametric distribution. Categorical variables were reported as absolute numbers and percentages, unless stated otherwise. Continuous numeric data were compared using the Student $t$ test or Mann-Whitney $\mathrm{U}$ test for parametric and non-parametric data, respectively. Categorical data were compared using the Chi-square or Fisher's Exact tests.

Statistical significance was assumed at $P<0.05$. KaplanMeier survival estimation was used to calculate stent patency rates. The statistical analyses were performed using SPSS statistical software version 25.0 (IBM Corp, Armonk, NY, USA).

\section{Results}

\section{Baseline Demographics}

87 patients with a median age of 63 (IQR 55-70) years were included. A total of 115 limbs were involved. As of June 2019, median follow-up time was 175 (IQR 57-257) days. Table 1 shows the baseline demographics. Forty-seven (54.0\%) were male, and 25 (28.7\%) patients had bilateral disease. All the patients were symptomatic with 43 (37.4\% of 115$)$ limbs having 
Table 1: Baseline Demographics

\begin{tabular}{|lc|}
\hline Variable & $\mathbf{N}=\mathbf{8 7}$ patients (\%) (IQR) (SD) \\
\hline Age, years & $62(43-82)$ \\
\hline Male & $47(54.0 \%)$ \\
\hline Duration of symptoms, months & $12(1-360)$ \\
\hline Bilateral venous disease & $25(28.7 \%)$ \\
\hline BMI & $28.50(\mathrm{SD} \pm 6.31)$ \\
\hline Diabetes mellitus & $32(36.8 \%)$ \\
\hline Hypertension & $66(75.9 \%)$ \\
\hline Hyperlipidemia & $65(74.7 \%)$ \\
\hline Peripheral vascular disease & $17(19.5 \%)$ \\
\hline Obstructive sleep apnea & $11(12.6 \%)$ \\
\hline Coagulation disorder & $7(8.0 \%)$ \\
\hline Previous DVT & $16(18.4 \%)$ \\
\hline Previous pulmonary embolism & $1(1.1 \%)$ \\
\hline History of smoking & $26(29.9 \%)$ \\
\hline
\end{tabular}

CEAP 6 disease. The median duration of symptoms was 12 (IQR 7-60) months. The median body mass index (BMI) was $28.50 \mathrm{~kg} / \mathrm{m}^{2}(\mathrm{SD} \pm 6.31)$ and only $7(8.0 \%)$ patients had a pre-existing known coagulation disorder. These patients required lifelong anti-coagulation. Seventy-nine (68.7\%) limbs had varicose veins with the majority of them (76) having had previous superficial venous ablation. Pre-operative DUS showed deep venous reflux in $63(54.8 \%)$ limbs and CTV showed compression in only $36(31.3 \%)$ limbs.

\section{Lesion Characteristics}

115 limbs were analysed (left $=76(66.1 \%))$. The majority of lesions were due to NIVL (97 (84.3\%)); the remainder comprised MTS (55 (47.8\%)) and PTS $(18(15.7 \%))$. The majority of limbs (66 (57.4\%)) had both CIV and EIV lesions. Thrombi were found in only $2(1.7 \%)$ limbs. Both patients with thrombi had an underlying coagulopathy disorder (anti-phospholipid syndrome in 1, and protein $\mathrm{S}$ deficiency in the other). Table 2 shows the lesion characteristics for all the patients.

\section{Procedural Characteristics and Complications}

173 stents were placed in 115 limbs (Sinus Obliquus $^{\mathrm{TM}}=63 / 173(36.4 \%) ;$ Venovo $^{\mathrm{TM}}=46 / 173$ $(26.6 \%)$; Wallstent ${ }^{\mathrm{TM}}=63 / 173(36.4 \%)$; Zilver
Table 2: Lesion Characteristics

\begin{tabular}{|lc|}
\hline Lesion Characteristics & N $=115$ limbs $(\%)$ \\
\hline Left limb & $76(66.1 \%)$ \\
\hline NIVL & $97(84.3 \%)$ \\
\hline MTS & $55(47.8 \%)$ \\
\hline Presence of varicose veins & $79(68.7 \%)$ \\
\hline Pre-op. CTV showing compression & $36(31.3 \%)$ \\
\hline Deep venous reflux on DUS & $63(54.8 \%)$ \\
\hline Previous superficial venous ablation & $76(66.1 \%)$ \\
\hline Previous trauma / surgery & $76(66.1 \%)$ \\
\hline CEAP class & \\
\hline CEAP 3 & $3(2.6 \%)$ \\
\hline CEAP 4a & $18(15.7 \%)$ \\
\hline CEAP 4b & $36(31.3 \%)$ \\
\hline CEAP 5 & $15(13.0 \%)$ \\
\hline CEAP 6 & $43(37.4 \%)$ \\
\hline Isolated CIV lesions & $29(25.2 \%)$ \\
\hline Isolated EIV lesions & $13(11.3 \%)$ \\
\hline CIV and EIV lesions & $66(57.4 \%)$ \\
\hline CIV, EIV and CFV lesions & $7(6.1 \%)$ \\
\hline & $2(1.7 \%)$ \\
\hline Presence of thrombus & $2 \%)$ \\
\hline Prence of occlusion & \\
\hline
\end{tabular}

Vena $\left.^{\text {TM }}=1 / 173(0.6 \%)\right)$. The mean operative time was $77.5(\mathrm{SD} \pm 40.4)$ minutes. A median of 1 (IQR 1 - 2) stent was placed per limb. A combination of Sinus Obliquus ${ }^{\mathrm{TM}}$ and Venovo ${ }^{\mathrm{TM}}$ stents was deployed in $23(20.0 \%)$ limbs. A combination of Sinus Obliquus $^{\mathrm{TM}}$ and Wallstent ${ }^{\mathrm{TM}}$ was deployed in $12(10.4 \%)$ limbs. Pre-dilatation was employed in all the procedures, and all achieved technical and procedural success. Rivaroxaban (Bayer AG, Leverkusen, Germany) was the post-operative anti-coagulation of choice in $93 / 115$ $(80.9 \%)$ procedures (Warfarin $=19 / 115(16.5 \%)$; Dual anti-platelet therapy (DAPT) $=2 / 115(1.7 \%)$; Apixaban $=1 / 115(0.9 \%))$. The median length of hospitalisation was 1 day (IQR 1 - 2). 16/115 $(13.9 \%)$ procedures had post-operative complications (Post-operative fever $=5 / 16(31.3 \%)$; asymptomatic bradycardia $=1 / 16(6.3 \%)$; cellulitis $=2 / 16(12.5 \%)$; in-stent thrombosis $=5 / 16(31.3 \%)$; in-stent stenosis $=3 / 16(18.8 \%))$. 


\section{Patency and Stent Integrity}

Overall primary patency rates were $98.2 \%(112 / 114)$, $97.9 \%(93 / 95), 95.7 \%(89 / 93)$ and $92.8 \%(64 / 69)$ immediately post-intervention, and at 3, 6 and 12 months respectively. 5/87 (5.7\%) patients developed instent thrombosis (Day 1 post-procedure $=2 / 5(40.0 \%)$; 6 months $=2 / 5(40.0 \%) ; 12$ months $=1 / 5(20 \%)) .2 / 5(40.0 \%)$ with in-stent thrombosis had PTS (NIVL $=3 / 5(60.0 \%))$. $1 / 5(20.0 \%)$ patient who developed in-stent thrombosis had underlying coagulopathy (Protein-S deficiency). $3 / 87(3.4 \%)$ patients developed in-stent restenosis. $6 / 8(75.0 \%)$ of patients who had in-stent restenosis or thrombosis underwent reintervention. One patient with in-stent thrombosis had a spontaneous resolution of thrombi. Another refused reintervention. Secondary patency rate was $99.1 \%(114 / 115)$ at 6 months. 5/8 $(62.5 \%)$ that developed loss of stent patency involved Wallstent ${ }^{\mathrm{TM}}$ usage. Figure 1 shows the Kaplan-Meier curves for the cumulative patency rates for PTS and NIVL.

\section{Clinical Outcomes}

The mean rVCSS decreased from 11.52 ( $\mathrm{SD} \pm 3.54$ ) at baseline to $5.77(\mathrm{SD} \pm 2.36)$ at 3 months $(P<0.01)$. $59 / 115(51.3 \%)$ had $350 \%$ rVCSS score reduction. $115 / 115(100 \%)$ limbs experienced ${ }^{32}$-point rVCSS score reduction. The mean VAS score decreased from $6.62(\mathrm{SD} \pm 1.93)$ to $2.92(\mathrm{SD} \pm 1.50)$ at 3 months $(P<0.01) .2 / 115(1.7 \%)$ had no improvement in VAS pain score. One of these patients had no pain (VAS score $=0$ ) at baseline. No patient experienced an increase in VAS pain score. 41/43 (95.3\%) venous ulcers healed completely over a median time of 169 days (IQR 120-253). Of the 2 patients who did not experience ulcer healing, 1 had in-stent restenosis detected on DUS at 12 months post-intervention and was re-intervened on but still has a small chronic wound. Figure 2 shows the clinical improvement of a patient with bilateral CEAP 6 disease 6 months post-intervention.

\section{Discussion}

At our centres, an overwhelming majority of the limbs $(84.3 \%)$ presented with NIVLs rather than PTS, despite previously reported increased frequency rates of acute DVT in Singapore. ${ }^{24}$ Most existing studies report a lower proportion of NIVLs. A study conducted by Raju et al reported that slightly more than half $(53.0 \%)$ of IFVO were NIVLs. ${ }^{25}$ A smaller study of 200 patients reported just under half of them $(48.5 \%)$ had NIVLs. ${ }^{7}$ This is likely to be due to a local referral pathway issue. At our centres, PTS patients were initially managed by haematologists. Patients underwent trials of graduated external compression stockings, venoactive drugs and exercise training programmes. ${ }^{26}$ Only those with severe refractory venous insufficiency despite medical therapy were referred to vascular surgeons for evaluation.

In our study, nearly half (47.8\%) of the limbs had MTS. The true prevalence of MTS is unknown. It is estimated to be present in $2 \%$ to $5 \%$ of patients with lower limb venous disease. ${ }^{27-29}$ May and Thurner found spur-like projections in the left common iliac vein in $22 \%$ of 430 cadavers examined. ${ }^{2}$ MTS is underdiagnosed, possibly due to its diagnostic difficulty and permissive role in chronic venous disease. ${ }^{28}$ MTS is often asymptomatic, until an additional insult or pathology is superimposed, such as cellulitis or osteoarthritis of the knee resulting in loss of the venous calf pump mechanism. ${ }^{25,30}$ A small prospective study on 20 asymptomatic volunteers who underwent angiography of the iliac veins found that $80 \%$ had at least 2 signs indicative of $\mathrm{MTS}^{31}$ At our centres, asymptomatic patients with MTS did not undergo prophylactic IVUS and deep vein stenting.

All patients underwent DUS to rule out acute deep vein thrombosis and identify the presence of deep vein reflux. Just slightly more than half $(54.8 \%)$ of the limbs showed deep vein reflux on DUS, suggesting that deep vein reflux is not necessary for diagnosing IFVO. Pre-operative CTV or MRV was performed for all patients to rule out obvious extrinsic causes of compression such as tumours, fibroids or a distended bladder. CTV and MRV provided a clearer visualisation of anatomic compression sites and collaterals, which indicates the hemodynamic significance of the stenosis. ${ }^{32}$ However, IVUS is the gold standard for diagnosis of obstructive ilio-femoral venous lesions. ${ }^{33,34}$ Only 36 (31.3\%) limbs with IVUS-proven IFVO showed signs of compression on pre-operative CTV or MRV. The absence of compression on CTV or MRV does not exclude underlying IFVO. IVUS is also superior to multi-planar catheter-based venography (MPV) (Figure 3). The VIDIO trial reported that IVUS was significantly more sensitive than MPV in identifying and characterising venous lesions. ${ }^{35}$ IVUS triumphs MPV in predicting clinical improvement post-stenting. ${ }^{36}$ IVUS is also an invaluable aid in the accurate placement of venous stents after venoplasty, especially around the ilio-caval confluence. ${ }^{33}$

Short-term stent patency rates were favorable. Secondary patency rate was $99.1 \%$ at 6 months, with minimal reinterventions. PTS patients had lower stent patency rates compared to NIVL patients, consistent with existing studies. A meta-analysis of 1500 patients 


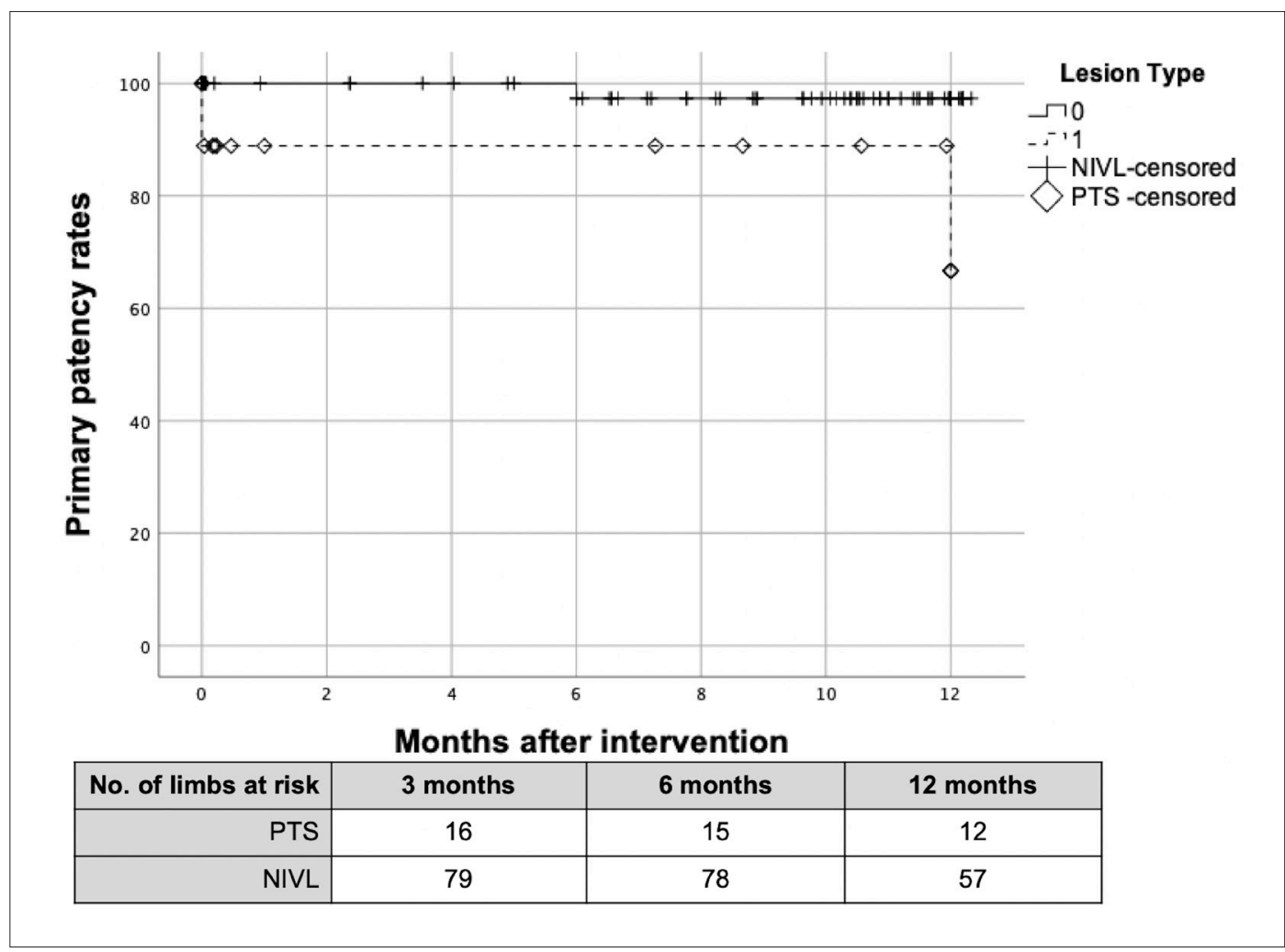

Fig. 1. Kaplan-Meier Curves for Stent Patency Rates in PTS vs NIVL Patients

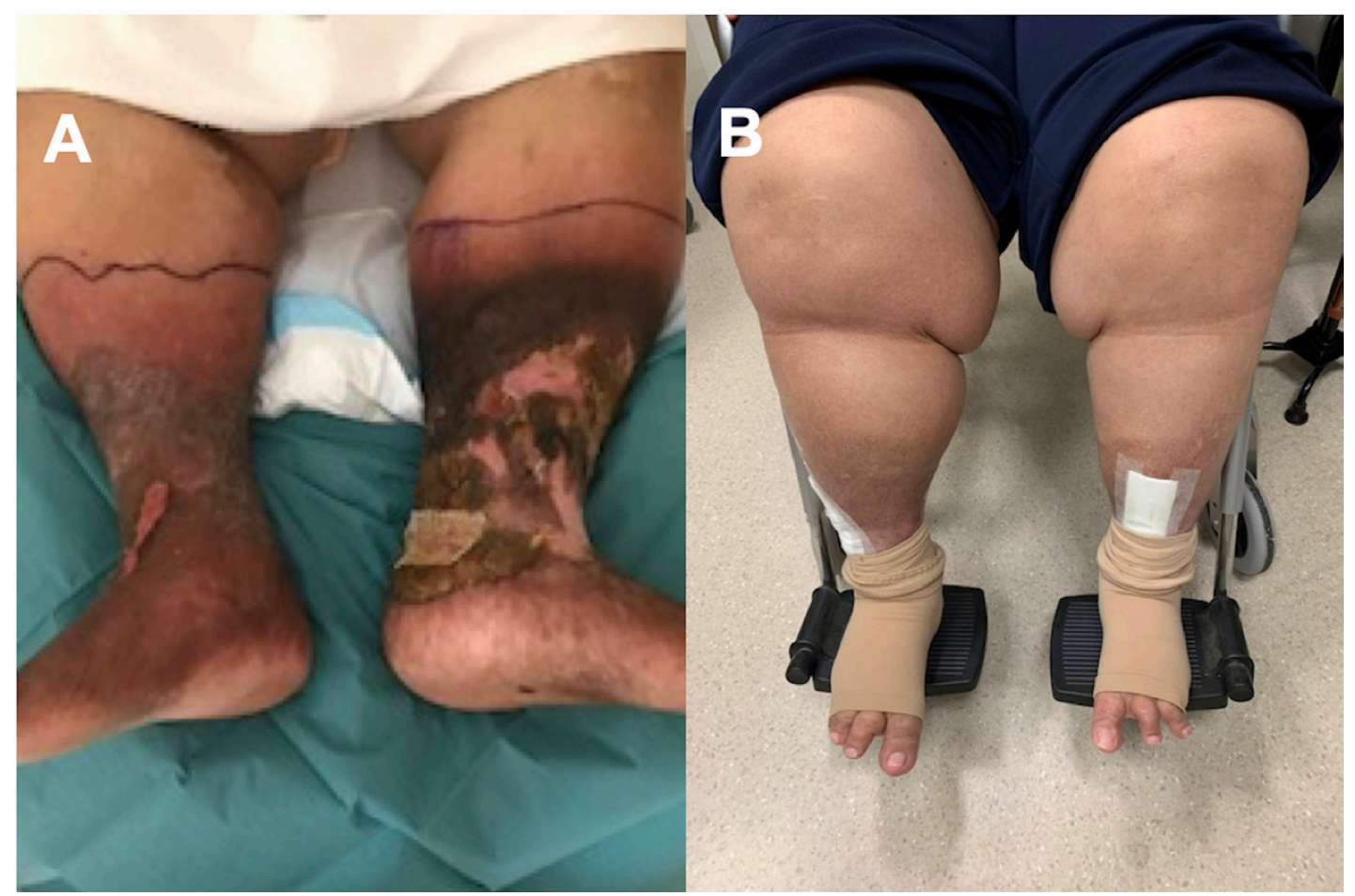

Fig. 2. Pre-operative and Post-operative Clinical Picture of a Patient with Bilateral CEAP 6 Venous Disease. A, Pre-operative clinical picture demonstrating bilateral limb swelling, diffused venous skin changes and venous stasis ulcers. B, Clinical picture of bilateral limbs 6 months post-operation, demonstrating significant alleviation of symptoms. 


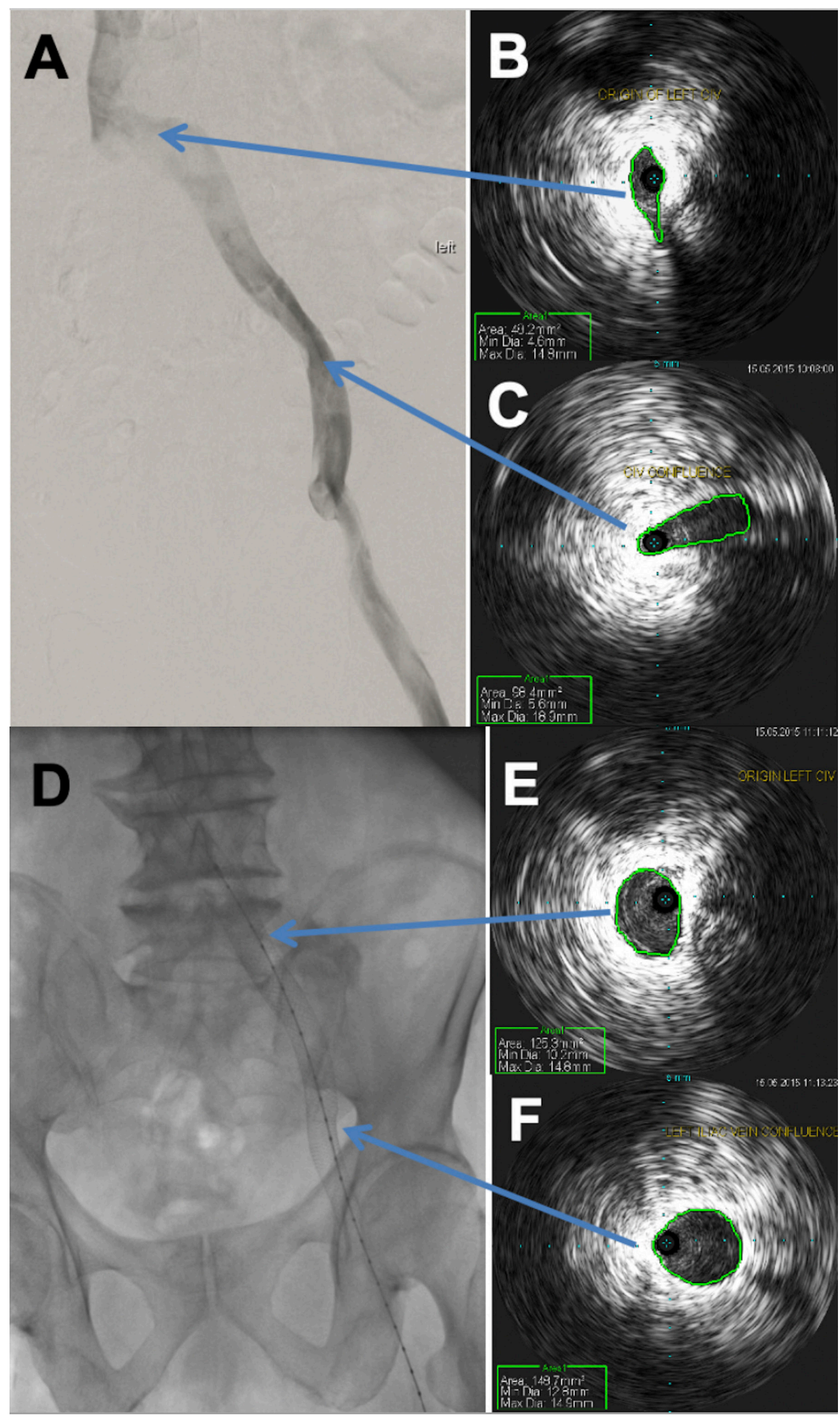

Fig. 3. Pre-operative and Post-operative Imaging of a Patient with Left Common Iliac Vein (Origin and Confluence) Lesions. A, Pre-operative venography demonstrating obstruction. B and C, Pre-operative IVUS demonstrating obstruction at origin and confluence of left common iliac vein respectively. Obstruction is better visualized on IVUS. D, Post-operative venography demonstrating a good result with no residual obstruction. E and F, Post-operative IVUS shows well expanded stent with adequate lumen at origin and confluence of left common iliac vein respectively. 
revealed stent patency rates of $90-100 \%$ for NIVLs compared to $74-89 \%$ for PTS patients. ${ }^{37}$ Contributing factors affecting stent patency rates include stents terminating below the inguinal ligament, long length of stents and presence of thrombophilia..$^{38}$ No stent fractures or stent migration were experienced in our series. Five out of $8(62.5 \%)$ instances of loss of stent patency involved Wallstent ${ }^{\mathrm{TM}}$ (Boston Scientific, Marlborough, USA). The Wallstent ${ }^{\mathrm{TM}}$ is not a dedicated venous stent. It has less radial force at its ends, and significant foreshortening during the post-dilatation process, making it difficult to predict final positioning especially near the ilio-caval junction. Medium term follow-up has shown compressive effects typically at the iliac confluence area. ${ }^{39}$ We have now stopped deploying the Wallstent ${ }^{\mathrm{TM}}$ for the last 3 years. Currently, Venovo ${ }^{\mathrm{TM}}$ (BD Medical, Arizona, USA) and Sinus Obliquus ${ }^{\mathrm{TM}}$ (Optimed, Ettlingen, Germany) are deployed as the stents of choice.

The Venovo ${ }^{\mathrm{TM}}$ venous stent system comprises a selfexpandable nitinol stent that is uniquely flexible for venous vessels, with flared ends to ensure adequate wall apposition. ${ }^{40}$ Sinus Obliquus ${ }^{\mathrm{TM}}$ is the first oblique venous stent, specifically designed for treating venous obstructions close to the bifurcation of the IVC, such as in MTS. The oblique design enables the stent to be placed directly at the bifurcation. Similar to our experience, data from the Arnsberg venous registry revealed favourable short-term patency rates for both stents. Primary and secondary patency rates were $97 \%$ and $100 \%$ at 6 months post-intervention for Venono ${ }^{\mathrm{TM}}$ stents. ${ }^{40}$ Primary and secondary patency rates were $94 \%$ and $96 \%$ at 12 months post-intervention for Sinus Obliquus $^{\mathrm{TM}}$ stents. ${ }^{41}$ Both stents were associated with statistically significant improvement in clinical outcomes and low complications rates. The ongoing VERNACULAR trial on the Venovo ${ }^{\mathrm{TM}}$ stent also reported excellent primary patency of $83 \%$ rates at 24 months post-intervention, with no stent fractures. ${ }^{42}$

While there are innumerable studies reporting excellent patency rates and clinical outcomes following deep vein stenting for IFVO, the quality of evidence is low. ${ }^{43-45}$ Most available studies are small, retrospective, single-site evaluations. ${ }^{46} \mathrm{~A}$ systematic review conducted by Seager et al identified no randomised controlled trials, cohort studies or case-control studies. A GRADE assessment ${ }^{47}$ demonstrated the quality of the evidence to be "low" for ulcer healing and "very low" for other outcomes. ${ }^{44}$ Despite the weak quality of evidence, deep vein stenting is relatively effective and safe with low incidence of peri-operative complications and high patency rates, ${ }^{9,43-46}$ and should be considered as a treatment option while the evidence base improves. ${ }^{44}$
Post-operative anticoagulation decreases complication rates such as in-stent thrombosis. However, there is no official recommendation on the choice of anticoagulation and the duration of therapy post-procedure. ${ }^{10,48}$ Vitamin $\mathrm{K}$ Antagonists (VKA) are the most commonly used agents, but there is a major shift towards the use of Direct Oral Anticoagulants (DOACs) recently. ${ }^{49}$ At our centres, Rivaroxaban (Bayer AG, Leverkusen, Germany) was the anticoagulation of choice in 93/115 (80.9\%) procedures, due to its ease of use and low complication rates. Rivaroxaban has minor drug and food interactions, a wide therapeutic window, and no need for laboratory monitoring. ${ }^{50}$ Rivaroxaban has a lower incidence of major bleeding. At our centre, only 1 case of intra-cerebral bleed was observed. All PTS patients were prescribed warfarin. Anti-coagulation was discontinued and replaced with aspirin 6 months after stenting for NIVLs, if DUS surveillance was satisfactory. Patients with a history of recurrent DVTs or coagulation disorders usually maintain lifelong anticoagulation. Our practice is consistent with the international consensus achieved using Delphi methodology in the first-ever study investigating antithrombotic practices after venous stenting-52/61 (85.2\%) of the study respondents discontinued anticoagulation 6-12 months post-stenting for NIVLs. 51/60 (85.0\%) started lifelong anticoagulation for patients with recurrent DVT or coagulopathy. ${ }^{49}$

Thrombophilia workup is not routinely done at our centres. Only patients with a history of recurrent DVTs and pro-thrombotic events are screened for coagulation disorders because the pick-up rate is very low. While some studies have identified high markers of thrombophilia in $10.8 \%$ of patients presenting with DVT in a Caucasian based population, ${ }^{51}$ there is a lower incidence in Asian populations. A local study of 60 thromboembolic patients revealed that the incidence of resistance to activated protein C (APC-R), the most common inherited cause of thrombosis in Caucasians, is much lower in Singapore as compared to the West. ${ }^{52}$ A Taiwanese study revealed that mutations at position 20210 in the prothrombin gene, a form of inherited thrombophilia, is extremely rare in Chinese. ${ }^{53}$

\section{Conclusion}

In our series, the majority of patients requiring deep vein stenting presented with NIVLs rather than PTS, which may be a referral pathway issue. Deep vein stenting of symptomatic IFVO was associated with low complication rates and excellent short-term primary patency rates, with few re-interventions. There was marked improvement in clinical outcomes at 3 months, with a high and expedient venous ulcer healing rate. 
However, rigorous evaluation of safety and long-term effectiveness of deep vein stenting with novel dedicated venous stents is required. Further studies are necessary to optimise post-operative anticoagulation regimes.

\section{REFERENCES}

1. Kahn SR PH, Vedantham S, Prandoni P, Kearon C; Subcommittee on Control of Anticoagulation of the Scientific and Standardization Committee of the International Society on Thrombosis and Haemostasis. Definition of post-thrombotic syndrome of the leg for use in clinical investigations: a recommendation for standardization. Journal of Thrombosis and Haemostasis 2009;7(5):879-883.

2. R. May, Thurner J. The Cause of the Predominantly Sinistral Occurrence of Thrombosis of the Pelvic Veins Angiology 1957;8(5):419-427.

3. Du Pont B, Verbist J, Van den Eynde W, P P. Right-sided Cockett's syndrome. Acta Chir Belg. 2016 Apr;116(2):114-118.

4. Vijayalakshmi IB SH, Narasimhan C. Unusual cases of right-sided and left-sided May-Thurner syndrome. Cardiol Young. 2015 Apr; 25(4):797-799.

5. Rohr A, Maxwell K, Best S, Lemons S, Johnson P. Rare presentation and endovascular treatment of multifocal iliac venous stenoses due to right sided May-Thurner Syndrome. Radiol Case Rep. 2019 Dec 18;15(3):201-203.

6. Kahn SR. The post-thrombotic syndrome. Hematology Am Soc Hematol Educ Program. 2016 Dec 2;2016(1):413-418.

7. van Vuuren T, Doganci S, Wittens CHA. Patency rates and clinical outcomes in a cohort of 200 patients treated with a dedicated venous stent. J Vasc Surg Venous Lymphat Disord. 2018 May;6(3):321-329.

8. Neglen P, Berry MA, Raju S. Endovascular surgery in the treatment of chronic primary and post-thrombotic iliac vein obstruction. Eur J Vasc Endovasc Surg. 2000 Dec;20(6):560-571.

9. Wen-da W, Yu Z, Yue-Xin C. Stenting for chronic obstructive venous disease: A current comprehensive meta-analysis and systematic review. Phlebology. 2016 Jul;31(6):376-389.

10. StuckAK, Reich T, Engelberger RP, Sebastian T, Kucher N. Endovascular treatment of post-thrombotic and non-thrombotic iliofemoral venous outflow obstructions with self-expanding nitinol stents. Vasa. 2018 Jun;47(4):319-325.

11. Razavi M, Marston W, Black S, Bentley D, Neglen P. The initial report on 1-year outcomes of the feasibility study of the VENITI VICI VENOUS STENT in symptomatic iliofemoral venous obstruction. J Vasc Surg Venous Lymphat Disord. 2018 Mar;6(2):192-200.

12. Qian AM, Cai ZX, Zhang S, et al. Endovascular treatment for nonthrombotic right iliac vein compression syndrome with intravascular ultrasound. Zhonghua Yi Xue Za Zhi. 2019 Dec;99(46):3633-3637.

13. George R, Verma H, Ram B, Tripathi R. The effect of deep venous stenting on healing of lower limb venous ulcers. Eur J Vasc Endovasc Surg. 2014 Sep;48(3):330-336.

14. Huang C, Yu G, Huang J. Midterm Results of Endovascular Treatment for Iliac Vein Compression Syndrome from a Single Center. Ann Vasc Surg. 2018 May;49:57-63.

15. Kaichuang Ye, Xinwu Lu, Weimin Li, et al. Long-Term Outcomes of Stent Placement for Symptomatic Nonthrombotic Iliac Vein Compression Lesions in Chronic Venous Disease. Journal of vascular and interventional radiology: JVIR. 2012;23(4):497-502.

16. Liu Z, Gao N, Shen L, et al. Endovascular treatment for symptomatic iliac vein compression syndrome: a prospective consecutive series of 48 patients. Ann Vasc Surg. 2014 Apr;28(3):695-704.
17. Lou WS, Gu JP, He X, et al. Endovascular treatment for iliac vein compression syndrome: a comparison between the presence and absence of secondary thrombosis. Korean $J$ Radiol. Mar-Apr 2009;10(2):135-143.

18. Sang H, Li X, Qian A, Meng Q. Outcome of endovascular treatment in postthrombotic syndrome. Ann Vasc Surg. 2014 Aug;28(6):1493-1500.

19. Ye K. Technical Details and Clinical Outcomes of Transpopliteal Venous Stent Placement for Postthrombotic Chronic Total Occlusion of the Iliofemoral Vein. J Vas Interv Radiol. 2014 Jun;25(6):925-932.

20. QWS Lee KG, SL Chan, HP Rathnaweera, TT Chong, TY Tang. A comparison between Caucasian and Asian superficial venous anatomy and reflux patterns-Implications for potential precision endovenous ablation therapy. Phlebology. 2019;35(1):39-45.

21. Rutherford RB, Padberg FT, Jr., Comerota AJ, Kistner RL, Meissner MH, Moneta GL. Venous severity scoring: An adjunct to venous outcome assessment. J Vasc Surg. 2000 Jun;31(6):1307-1312.

22. Vasquez MA, Munschauer CE. Venous Clinical Severity Score and quality-of-life assessment tools: application to vein practice. Phlebology. 2008;23(6):259-275.

23. Aurshina A, Chait J, Kibrik P, et al. Efficacy of balloon venoplasty alone in the correction of nonthrombotic iliac vein lesions. J Vasc Surg Venous Lymphat Disord. 2019 Sep;7(5):665-669.

24. L H Lee, K Q Gu, Heng D. Deep Vein Thrombosis Is Not Rare in Asia--the Singapore General Hospital Experience. Ann Acad Med Singapore. 2002 Nov;31(6):761-764.

25. Raju S, Neglen P. High prevalence of nonthrombotic iliac vein lesions in chronic venous disease: a permissive role in pathogenicity. $J$ Vasc Surg. 2006 Jul;44(1):136-143.

26. Kahn SR, Comerota AJ, Cushman M, et al. The postthrombotic syndrome: evidence-based prevention, diagnosis, and treatment strategies: a scientific statement from the American Heart Association. Circulation. 2014 Oct;130(18):1636-1661.

27. Cavalcante LP, Souza JEdS, Pereira RM, et al. Iliac vein compression syndrome: literature review. Jornal Vascular Brasileiro. 2015;14(1):78-83.

28. Harbin MM, Lutsey PL. May-Thurner syndrome: History of understanding and need for defining population prevalence. $J$ Thromb Haemost. 2020 Mar;18(3):534-542.

29. Mousa AY, AbuRahma AF. May-Thurner syndrome: update and review. Ann Vasc Surg. 2013 Oct;27(7):984-995.

30. Radaideh Q, Patel NM, Shammas NW. Iliac vein compression: epidemiology, diagnosis and treatment. Vasc Health Risk Manag. 2019 May;15:115-122.

31. van Vuuren T, Kurstjens RLM, Wittens CHA, van Laanen JHH, de Graaf R. Illusory Angiographic Signs of Significant Iliac Vein Compression in Healthy Volunteers. Eur J Vasc Endovasc Surg. 2018 Dec;56(6):874-879.

32. Toh MR, Tang TY, Lim HHMN, Venkatanarasimha N, K D. Review of imaging and endovascular intervention of iliocaval venous compression syndrome. World Journal of Radiology. 2020 Mar;12(3):18-28.

33. Neglen P, Raju S. Intravascular ultrasound scan evaluation of the obstructed vein. J Vasc Surg. 2002 Apr;35(4):694-700.

34. MR Toh, K Damodharan, MNHH Lim, et al. Intravascular Ultrasound (IVUS) for Deep Venous Work - Want or Need? Journal of Cardiology and Cardiovascular Therapy 2020;16(2).

35. Gagne PJ, Tahara RW, Fastabend CP, et al. Venography versus intravascular ultrasound for diagnosing and treating iliofemoral vein obstruction.J Vasc Surg Venous Lymphat Disord. 2017 Sep;5(5):678-687. 
36. Gagne PJ, Gasparis A, Black S, et al. Analysis of threshold stenosis by multiplanar venogram and intravascular ultrasound examination for predicting clinical improvement after iliofemoral vein stenting in the VIDIO trial. J Vasc Surg Venous Lymphat Disord. 2018;6(1):48-56

37. Raju S. Best management options for chronic iliac vein stenosis and occlusion. J Vasc Surg. 2013 Apr;57(4):1163-1169.

38. Neglén P, Raju S. In-stent recurrent stenosis in stents placed in the lower extremity venous outflow tract. Journal of Vascular Surgery. 2004 Jan;39(1):181-187.

39. Gagne PJ, Gagne N, Kucher T, Thompson M, Bentley D. Long-term clinical outcomes and technical factors with the Wallstent for treatment of chronic iliofemoral venous obstruction. J Vasc Surg Venous Lymphat Disord. 2019 Jan;7(1):45-55.

40. Lichtenberg MKW, de Graaf R, Stahlhoff WF, Ozkapi A, Rassaf T, Breuckmann F. Venovo venous stent in the treatment of non-thrombotic or post-thrombotic iliac vein lesions - short-term results from the Arnsberg venous registry. Vasa. 2019 Mar;48(2):175-180.

41. Lichtenberg M dGR, Stahlhoff WF, Özkapi A, Simon M, Breuckmann F. Patency rates, safety and clinical results of the sinus-Obliquus venous stent in the treatment of chronic ilio-femoral venous outflow obstruction - data from the Arnsberg venous registry. VASA Zeitschrift fur Gefasskrankheiten. 2019;48(3):270-275.

42. BARD® The VENOVOTM Venous Stent Study for Treatment of Iliofemoral Occlusive Disease. https://ClinicalTrials.gov/show/ NCT02655887. Published 2016. Accessed on 20 December 2019.

43. Williams ZF, Dillavou ED. A systematic review of venous stents for iliac and venacaval occlusive disease. J Vasc Surg Venous Lymphat Disord. 2020 Jan;8(1):145-153.

44. Seager MJ, Busuttil A, Dharmarajah B, Davies AH. Editor's Choice-- A Systematic Review of Endovenous Stenting in Chronic Venous Disease Secondary to Iliac Vein Obstruction. Eur J Vasc Endovasc Surg. 2016 Jan;51(1):100-120.
45. Qiu P, Zha B, Xu A, et al. Systematic Review and Meta-Analysis of Iliofemoral Stenting for Post-thrombotic Syndrome. Eur J Vasc Endovasc Surg. 2019 Mar;57(3):407-416.

46. Razavi MK, Jaff MR, Miller LE. Safety and Effectiveness of Stent Placement for Iliofemoral Venous Outflow Obstruction: Systematic Review and Meta-Analysis. Circ Cardiovasc Interv. 2015 Oct;8(10):e002772.

47. Schünemann HJ, Oxman AD, Brozek J, et al. GRADE: assessing the quality of evidence for diagnostic recommendations. Evid Based Med. 2008 Dec;13(6):162-163.

48. Yap HY, Loa J, Tosenovsky PJ, Lee SQ, Chong TT, Tang TY. Use of anticoagulation therapy post deep venous stenting for post-thrombotic disease and non-thrombotic iliac vein lesions - Any consensus? Phlebology. 2020 Jan:268355520901661

49. Milinis K, Thapar A, Shalhoub J, Davies AH. Antithrombotic Therapy Following Venous Stenting: International Delphi Consensus. Eur J Vasc Endovasc Surg. 2018 Apr;55(4):537-544

50. Mekaj YH, Mekaj AY, Duci SB, Miftari EI. New oral anticoagulants: their advantages and disadvantages compared with vitamin $\mathrm{K}$ antagonists in the prevention and treatment of patients with thromboembolic events. Ther Clin Risk Manag. 2015 Jun;11:967-977.

51. Caprini JA, Goldshteyn S, Glase CJ, Hathaway K. Thrombophilia testing in patients with venous thrombosis. Eur J Vasc Endovasc Surg. 2005 Nov;30(5):550-555.

52. Lim LC TH, Lee LH, Tien SL, Abdul Ghafar A. Activated protein C resistance: a study among 60 thromboembolic patients in the Singapore population. Annals of the Academy of Medicine, Singapore. 1999 Mar;28(2):252-255.

53. Lin J S SMC, Tsay W. The mutation at position 20210 in the 3'-untranslated region of the prothrombin gene is extremely rare in Taiwanese Chinese patients with venous thrombophilia. Thrombo Haemos. 1998 Aug;80(2):343. 